\title{
Kondo Hybridization and the Origin of Metallic States at the (001) Surface of $\mathrm{SmB}_{6}$
}

\author{
E. Frantzeskakis, ${ }^{1, *}$ N. de Jong, ${ }^{1}$ B. Zwartsenberg, ${ }^{1}$ Y. K. Huang, ${ }^{1}$ Y. Pan, ${ }^{1}$ X. Zhang, ${ }^{2}$ J. X. Zhang, ${ }^{2}$ F. X. Zhang, ${ }^{2}$ \\ L. H. Bao, ${ }^{3}$ O. Tegus, ${ }^{3}$ A. Varykhalov, ${ }^{4}$ A. de Visser, ${ }^{1}$ and M. S. Golden ${ }^{1, \dagger}$ \\ ${ }^{1}$ Van der Waals-Zeeman Institute, University of Amsterdam, Science Park 904, 1098 XH, Amsterdam, Netherlands \\ ${ }^{2}$ Key Laboratory of Advanced Functional Materials, Ministry of Education, College of Materials Science and Engineering, \\ Beijing University of Technology, Beijing 100124, China \\ ${ }^{3}$ Inner Mongolia Key Laboratory for Physics and Chemistry of Functional Materials, \\ Inner Mongolia Normal University, Hohhot 010022, China \\ ${ }^{4}$ Helmholtz-Zentrum Berlin für Materialien und Energie, Albert-Einstein-Strasse 15, 12489 Berlin, Germany
} (Received 12 August 2013; published 9 December 2013)

\begin{abstract}
$\mathrm{SmB}_{6}$, a well-known Kondo insulator, has been proposed to be an ideal topological insulator with states of topological character located in a clean, bulk electronic gap, namely, the Kondo-hybridization gap. Since the Kondo gap arises from many-body electronic correlations, $\mathrm{SmB}_{6}$ would be placed at the head of a new material class: topological Kondo insulators. Here, for the first time, we show that the $k$-space characteristics of the Kondo-hybridization process is the key to unraveling the origin of the two types of metallic states experimentally observed by angle-resolved photoelectron spectroscopy (ARPES) in the electronic band structure of $\mathrm{SmB}_{6}(001)$. One group of these states is essentially of bulk origin and cuts the Fermi level due to the position of the chemical potential $20 \mathrm{meV}$ above the lowest-lying $5 d-4 f$ hybridization zone. The other metallic state is more enigmatic, being weak in intensity, but represents a good candidate for a topological surface state. However, before this claim can be substantiated by an unequivocal measurement of its massless dispersion relation, our data raise the bar in terms of the ARPES resolution required, as we show there to be a strong renormalization of the hybridization gaps by a factor 2-3 compared to theory, following from the knowledge of the true position of the chemical potential and a careful comparison with the predictions from recent local-density-approximation (LDA) + Gutzwiller calculations. All in all, these key pieces of evidence act as triangulation markers, providing a detailed description of the electronic landscape in $\mathrm{SmB}_{6}$ and pointing the way for future, ultrahigh-resolution ARPES experiments to achieve a direct measurement of the Dirac cones in the first topological Kondo insulator.
\end{abstract}

DOI: 10.1103/PhysRevX.3.041024

\section{INTRODUCTION}

Kondo insulators have attracted enormous scientific interest, as they present a vivid manifestation of strong electronic correlations that give rise to exotic ground states in real materials [1-4]. They are based on elements with a partially filled $f$ shell. In such systems, the magnetic moment of localized $f$ electrons can be effectively screened by delocalized conduction carriers (i.e., $c-f$ interaction) leading to a hybridization-gap opening: the fingerprint of Kondo behavior in the electronic band structure. In many respects, $\mathrm{SmB}_{6}$ is a typical Kondo insulator [5-9], except that its residual resistivity at low temperatures is incompatible with the Kondo scenario [10-15].

Interest in $\mathrm{SmB}_{6}$ has recently been rekindled since bandstructure calculations [16-18] and transport measurements

\footnotetext{
*e.frantzeskakis@uva.nl

†m.s.golden@uva.nl
}

Published by the American Physical Society under the terms of the Creative Commons Attribution 3.0 License. Further distribution of this work must maintain attribution to the author(s) and the published article's title, journal citation, and DOI.
Subject Areas: Condensed Matter Physics

[19-23] have led to the proposition that electronic states of topological character might exist within the hybridization gap and be responsible for the nondivergent behavior of the resistivity at low temperatures. If this scenario is correct, not only is the long-standing challenge of understanding $\mathrm{SmB}_{6}$ 's behavior at low temperature solved, but also the first member of a new class of novel states of matter-topological Kondo insulators (TKIs) [16]—has been discovered. More than just representing a further "stamp" in the growing collection of novel quantum states of (electronic) matter, $\mathrm{SmB}_{6}$ could represent a very promising alternative to the Bi-based 3D topological insulators (TIs). In theory, Bi-based 3D TIs are systems with a bulk electronic gap at the Fermi level and conducting surface states, topologically protected from backscattering through a chiral spin texture [24]. Such compounds are promising for a wide spectrum of applications ranging from spintronics [25-27] to quantum computation [28]. However, in real crystals of these Bi-based (and related) 3D TIs, the effect of topologically trivial, transport-active defect states in the bulk gap [29] often masks the signatures of the topological surface states in transport experiments, and at 
present, this situation effectively prevents their exploitation in devices.

First, encouraging signs are emerging of charge transport involving topologically nontrivial states in $\mathrm{SmB}_{6}$ $[20,21]$, making further experimental investigation of the electronic structure of this system imperative.

As the hallmark of (strong) topological behavior is the observation of an odd number of Dirac cones in the electronic band structure (and their special spin texture), angle-resolved photoelectron spectroscopy (ARPES) has played a central role in the experimental investigation of 3D TIs to date [24]. Intriguingly, recent ARPES studies on the (001) cleavage surface of $\mathrm{SmB}_{6}$ crystals [30-33] have reported metallic states but failed to clearly identify a Dirac cone, thus leaving the question of the topological origin of the observed states unanswered.

Here, we present clear experimental evidence from ARPES on high-quality floating-zone-grown $\mathrm{SmB}_{6}$ crystals for two different metallic states in this system, and we provide a self-consistent and robust explanation of the origin of both these groups of metallic states, which rests on the cornerstone of the Kondo-insulator behavior of $\mathrm{SmB}_{6}$ : the $d-f$ hybridization. Our ARPES data provide three vital triangulation points for understanding the electronic structure of $\mathrm{SmB}_{6}$ crystals. First, a complete determination of the bulk $5 d$-related bands, the $4 f$-related features, and their hybridization at energy scales from the order of $1 \mathrm{eV}$ to a few tens of meV is provided. Second and third, within this framework, we succeed in an unambiguous identification of both the position of the chemical potential and the magnitude of the hybridization gaps in the low-lying electronic structure. Importantly, we discover that both of these experimentally determined characteristics of the near-surface electronic states of $\mathrm{SmB}_{6}$ are - as yet - incorrectly described by theory.

Combining the three key experimental ingredients from our ARPES data, we are able to pinpoint the exact locations in the $E(k)$ landscape of $\mathrm{SmB}_{6}$ in which very small hybridization gaps of 5-10 meV occur. It is in these gapswhich are not located at the Fermi energy of cleaved $\mathrm{SmB}_{6}$-in which ultrahigh-resolution ARPES investigations will have to search for the elusive Dirac cones that will prove the topological character of the Kondoinsulating behavior in this material. It is clear that the insight won here generates a well-defined and stringent framework, in which all future $k$-sensitive investigations of $\mathrm{SmB}_{6}$ will take their place.

\section{RESULTS AND DISCUSSION}

The transport data shown in Fig. 1(a) attest to the very high quality of the flux-free single crystals studied. The resistivity displays a sharp increase starting at $T \sim 50 \mathrm{~K}$, as in previous studies $[5,10,11,32]$, with saturation observed for $T<5 \mathrm{~K}$. The negative Hall resistivity in the saturation regime points toward electrons as the main carrier channel
$[10,11,13]$. The low-energy electron-diffraction (LEED) pattern of Fig. 1(b) proves the highly ordered, long-range single crystallinity of the cleavage surface, with sharp $1 \times 1$ spots evident over a large incident-electron-energy range (60-300 eV). This LEED pattern-observed for all cleaves of our crystals - is characteristic of high-quality $\mathrm{SmB}_{6}(001)$ surfaces, free from complicating surface reconstructions [34] whose effect in $k$ space is to obscure the fine details of the near- $E_{F}$ electronic band structure $[30,33]$ that are so crucial to the physics at hand. Figure 1(c) shows a schematic of the $\mathrm{SmB}_{6}$ Brillouin zone and its two-dimensional (2D) projection onto the (001) plane.

Figure 1(d) shows the backdrop for the remainder of the action presented in this paper, in the form of a complete $E\left(k_{x}, k_{y}\right)$ data set recorded from $\mathrm{SmB}_{6}$. These data have been acquired with $h \nu=70 \mathrm{eV}$, corresponding to a $k_{z}$ value of $6 \pi / a$ (see Figure S1), meaning the high-symmetry directions probed in the bulk Brillouin zone would be $\Gamma X$ and $X M$, as in the lower, green plane in Fig. 1(c).

In Fig. 1(d), three essentially nondispersive bands can be easily identified at binding energies $\left(E_{B}\right)$ of 40, 170, and $960 \mathrm{meV}$. The nondispersive bands originate from the Sm $4 f$ states, attributed to the ${ }^{6} \mathrm{H}_{5 / 2},{ }^{6} \mathrm{H}_{7 / 2}$, and ${ }^{6} \mathrm{~F}$ final-state multiplets of the $4 f^{6} \rightarrow 4 f^{5}$ transitions, respectively $[6,30,31,35]$. In addition, these flat bands are crossed by highly dispersive electronic states. First-principles calculations predict that the flat states have a dominant Sm $5 d$ character, with mixed $d-4 f$ character in energy regions (which we refer to later as hybridization zones) in which there is a strong interaction with the $4 f$ states [17,18,31].

Already in the data block of Fig. 1(d), these light $5 d$-related states can be seen to form elliptical constantenergy contours when not in the hybridization zones. Figures 2(a)-2(e) show how these elliptical contours grow steadily in size as $E_{B}$ is reduced, leading to an electron pocket centered on the $\bar{X}$ points of the (001)-projected Brillouin zone covering an area of $33 \%$ for $E=E_{F}$. These elliptical-contour electronic states are the first important group of states that cross $E_{F}$ in $\mathrm{SmB}_{6}$ and will be referred to as the $X$ states in the following.

In Figs. 2(1)-2(o), a zoom around the bulk $X$ point illustrates the evolution of the $X$ states. An elliptical guide to the eye has been added on top of the data for $E_{B}=430 \mathrm{meV}$, and this same guide has been added to Figs. 2(1), 2(m), and 2(o). The growth of the elliptical $X$ state from $1311 \mathrm{meV}$ [Fig. 2(o)] to $430 \mathrm{meV}$ [Fig. 2(n)] is evident, and for 77-meV binding energy [Fig. 2(m)], the elliptical-intensity contour in the data is, as expected, larger than the $E_{B}=430 \mathrm{meV}$ schematic dashed ellipse. Interestingly, the data for $10 \mathrm{meV}$ below the Fermi level show the $X$-state ellipse to be of the same size as $E_{B}=430 \mathrm{meV}$.

Figures 2(f)-2(h) provide the explanation for this renormalization of the $X$-state ellipses: In the hybridization zones-when the $5 d$-derived states cross the flat $4 f$ 


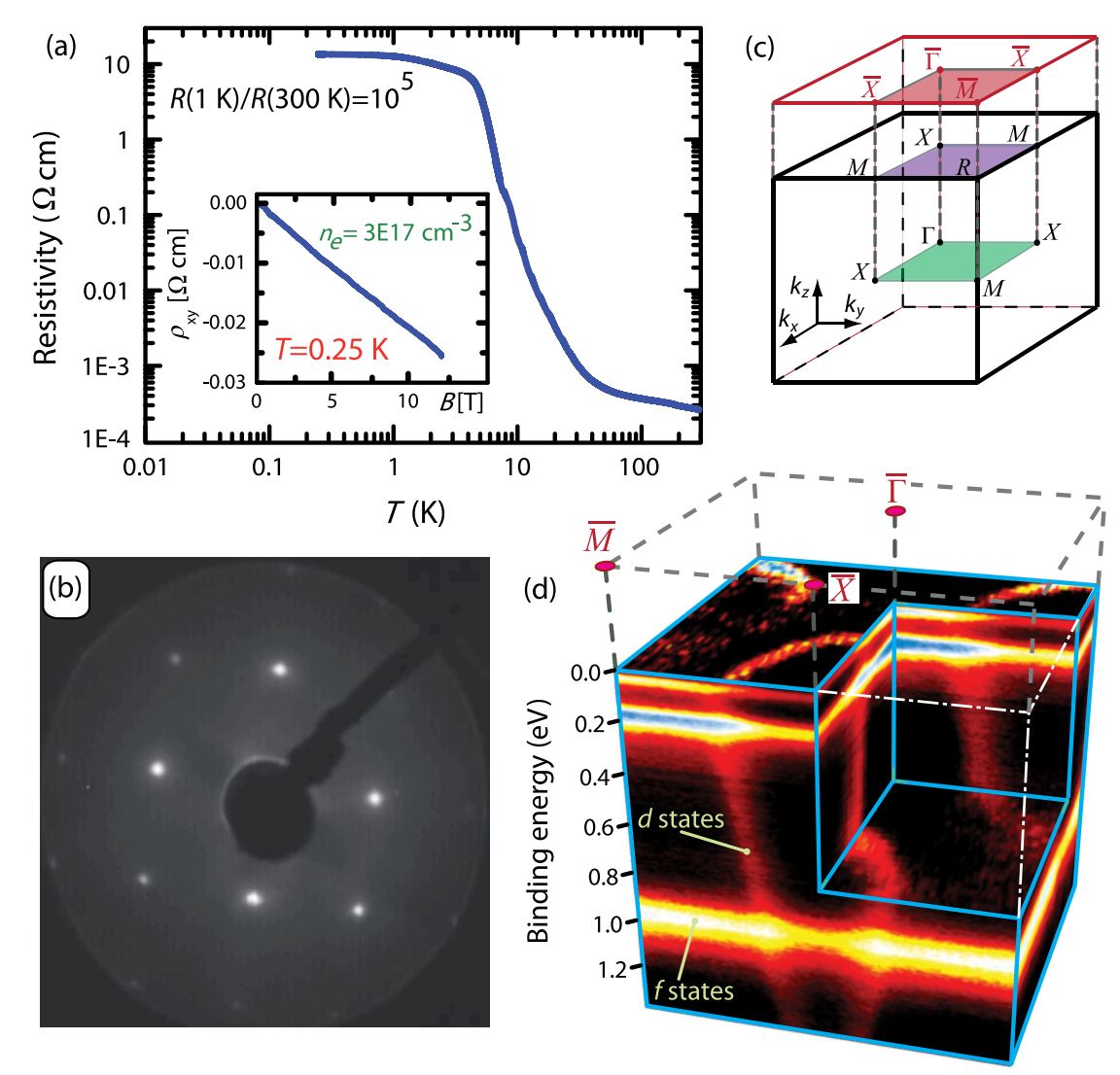

FIG. 1. $\mathrm{SmB}_{6}$ transport, surface quality, and ARPES data overview. (a) Temperature dependence of the resistivity of a floating-zone, optical-furnace-grown $\mathrm{SmB}_{6}$ crystal. There is a sharp increase below $50 \mathrm{~K}$ that results in a very high resistivity ratio of $10^{5}$ between the saturation regime and room temperature. The inset shows the Hall resistivity, which is linear, yielding an $n$-type carrier concentration as indicated (low $T$ regime). (b) Very sharp LEED spots reflect the simple $(1 \times 1)$ surface structure-free of reconstructions-and attest to the long-range order of the cleavage surface. The LEED primary beam energy is $97 \mathrm{eV}$. (c) Sketch of the 3D Brillouin zone of $\mathrm{SmB}_{6}$ and its projection onto the (001) plane. The colored planes denote the relevant cuts through the zone that would be made for photon energies in ARPES corresponding to a $k_{z}$ value of an even number of $\pi / a$ (green plane), an odd number of $\pi / a$ (purple plane), and the surface projection (red plane). (d) Three-dimensional ARPES data block displaying $I\left(E_{B}, k_{x}, k_{y}\right)$. Two types of bands are seen with the strongly dispersing (flat) bands originating from Sm $5 d(4 f)$ states, as indicated. The $\mathrm{Sm} 5 d$-related states give rise to elliptical contours at higher binding energies (the cutaway in the lower-right corner of the data block showing contours for a binding energy of $0.8 \mathrm{eV}$ ), which then evolve to give elliptical Fermi-surface contours around the $\bar{X}$ points. These states are discussed in the text as the $X$ states and are argued to be essentially bulk-derived bands, well described by bulk, ab initio theoretical approaches. Where the flat $4 f$ bands intersect the $5 d$-dominated $X$ states, complex band structures result because of the hybridization effects between $d$ and $f$ states of the equal symmetry, as will be discussed in detail in the context of Fig. 2. The photon energy for the data block is $h \nu=70 \mathrm{eV}$.

bands-the momentum distribution of the electronic states is altered radically. In Fig. 2(i), a schematic of the new energy contour is overlaid in yellow on the $E_{B}=40 \mathrm{meV}$ data, and Fig. 2(j) shows that the identical contour (now in blue) perfectly describes the experimental intensity distribution at $E_{B}=170 \mathrm{meV}$. Comparing the hybridization-zone energy contours and the $X$-state ellipses, as is done in Fig. 2(k), reveals the essentially fourfoldsymmetric fingerprint of the $d-f$ hybridization in $k_{x, y}$ space, which our data pick up in both the first and second Brillouin zones. We point out that in the hybridization zones themselves, the experimental intensity is high at the center of the Brillouin zone [see Figs. 2(f) and 2(g)]. The increased experimental intensity at hybridization zones and the equivalence of the $k$-space fingerprint of the hybridization at high energies $(170 \mathrm{meV})$ and low energies $(40 \mathrm{meV})$ is an important point and will be returned to later when discussing the electronic structure at and close to the Fermi level in more detail.

Closing our discussion of Fig. 2, we note that a consequence of the $d-f$ hybridization is that for $E_{B}$ a little lower than the hybridization zone [e.g., $E_{B}=10 \mathrm{meV}$ in Fig. 2(1)], although the elliptical shape of the $X$ states is reasserting itself, the $k$ value for these $5 d$-related states is reduced, compared to before the hybridization zone (see also the data movies in Figs. S2, S2a, and S2b of the Supplemental Material [36]). These phenomena-both in the hybridization zones as well as in the interaction-free energy windows - are in full agreement with data from bulk local-density-approximation (LDA), generalized- 


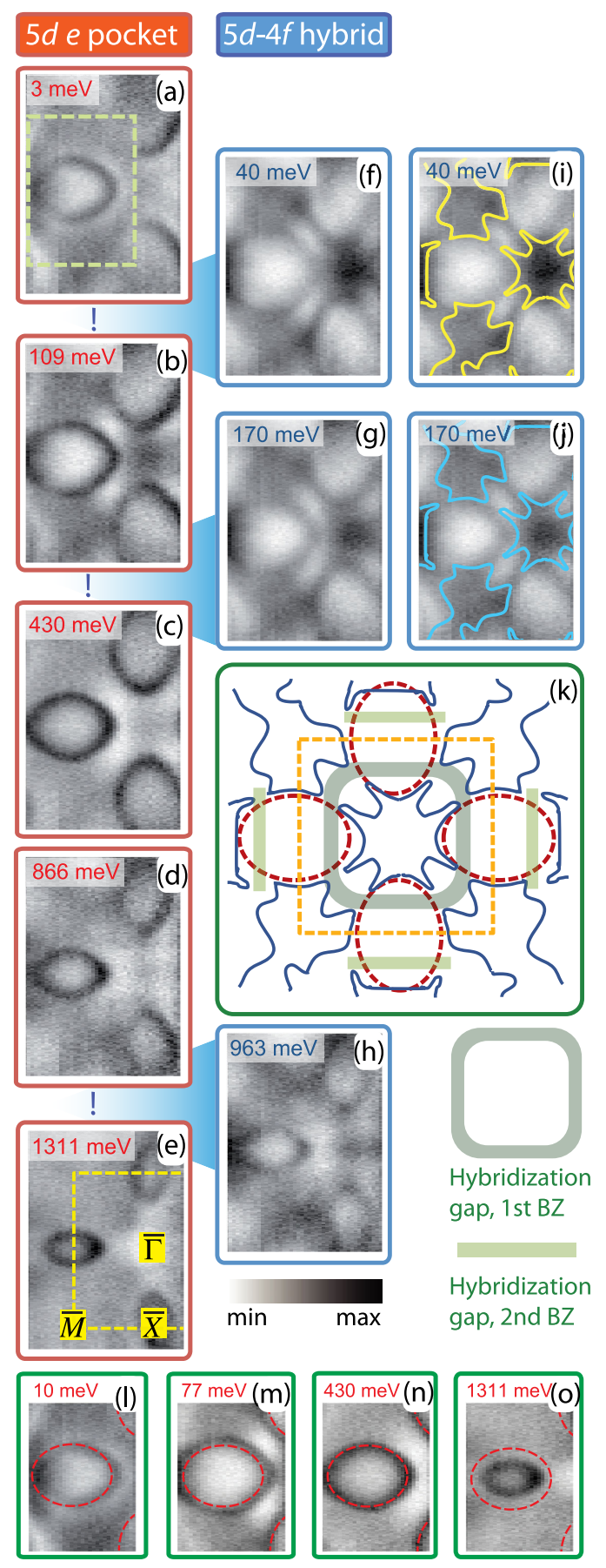

FIG. 2. $k$-space fingerprint of $5 d-4 f$ hybridization. (a)-(e) The elliptical contours from Sm $5 d$-dominated states centered at $\bar{X}$ increase in size as the binding energy decreases. The dashed yellow square in (e) shows the surface Brillouin zone (BZ). (f)-(h) When the $d$ and $f$ states interact strongly_-in hybridization zones—strong spectral weight redistribution occurs, giving significant intensity at $\bar{\Gamma}$. In (i), the constant-energy contours within the lowest-lying hybridization zone are indicated with yellow lines. (j) These same lines (now in blue) are also an excellent fit to the energy contours of the deeper-lying hybridization zone. (k) Exemplary hybridized (solid blue) and nonhybridized (dashed red) contours are overlaid. The broad, green-shaded lines highlight the resulting fourfold-symmetric $k$-space fingerprint of the $5 d-4 f$ hybridization, characterized by a gapping out of the ARPES intensity underlying parts of the dashed red ellipses. (1)-(o) Zooms of data covering the $k$-space area indicated with a dashed green box in (a). In (n), dashed red ellipses are drawn to match the data at 430-meV binding energy and the same ellipses are copied onto the other topmost panels, reiterating the trend that is also evident in (a)-(e) and in Fig. 1(d) that the $X$-state ellipses grow in size as the binding energy is increased. (l) Just after a hybridzation zone, the $X$-state ellipses shrink again, which leads to a $k$ discontinuity for these states. This second characteristic sign of strong 5d-4f interaction is also shown in Fig. 3 and discussed in detail in the context of Fig. 4. The ARPES data are acquired with $h \nu=70 \mathrm{eV}$. 
gradient-approximation, and LDA + Gutzwiller calculations, all of which predict states of mixed $d-f$ character upon interaction of the Sm $4 f$ and $\operatorname{Sm} 5 d$ states [17,18,31]. The calculated mixed $d-f$ character brings with it the inescapable conclusion that the altered $k$-space distribution and the discontinuity in the $X$-state $k$ values in the hybridization zones are nothing other than the signature of bulk $d-f$ hybridization. Occam's razor excludes an explanation going beyond the bulk physics of the hybridization itself, meaning the $X$-state $k$ renormalization cannot be interpreted as a consequence of band inversion and the occurrence of topologically protected electronic edge states.

We now turn to Fig. 3(a), in which an $E(k)$ image for the $\overline{M X M}$ direction, a zoom, and the momentum-distribution curve for $E=E_{F}$ are shown for $h \nu=70 \mathrm{eV}$ data, with the analogous cut along $\overline{X \Gamma X}$ shown in Fig. 3(b). The location of these cuts in $k$ space is indicated with the orange and purple arrows underlying the raw-data Fermi-surface map shown between Figs. 3(a) and 3(b).

At variance with theoretical calculations predicting a Kondo-hybridization gap at the Fermi level, the data of Fig. 3 show the sample to have a robustly metallic character. The zoom-in of Fig. 3(a) clearly shows the elliptical $X$ states crossing $E=E_{F}$, states that are also clearly identifiable in the data of both Figs. 1(d) and 2(a). In Fig. 3(b), however, the $E=E_{F}$ momentum-distribution curve clearly picks up a third maximum, located at $k=0$, in between the $X$-state peaks, indicating the existence of a second type of metallic state in $\mathrm{SmB}_{6}$, to which we forthwith refer as the $\Gamma$ state. We will return to the low-energy dispersions visible in the
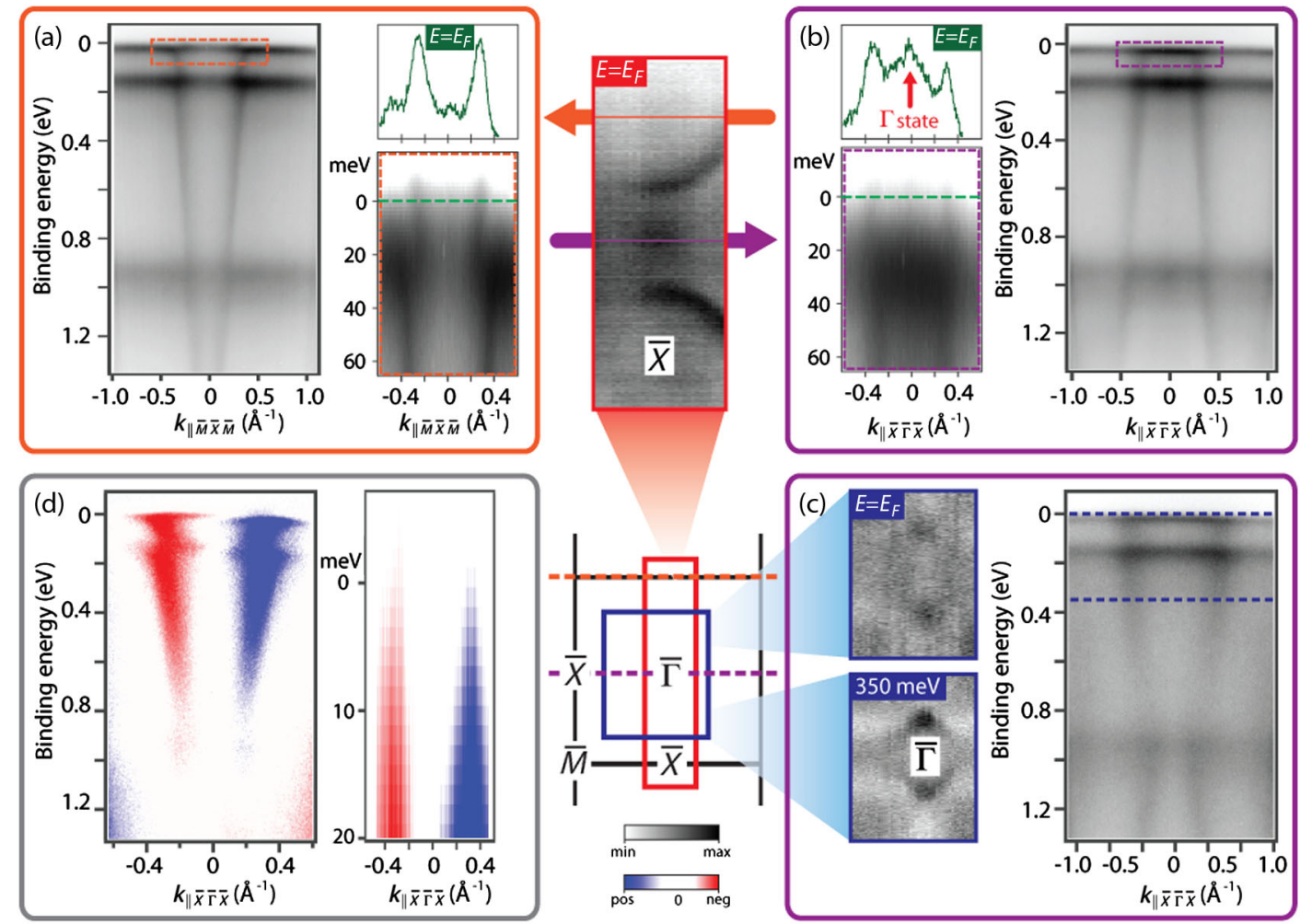

FIG. 3. Energy dispersion and dichroic signature of the $X$ state and identification of an additional $\Gamma$-state feature. (a),(b) Data recorded for $k_{z}=6 \pi / a(h \nu=70 \mathrm{eV})$ for the $k$-space cuts along the $\overline{M X M}$ and $\bar{X} \Gamma \bar{X}$ high-symmetry directions as indicated by the color-coded arrows underlying the Fermi-surface map in between the panels (integrated over $\pm 5 \mathrm{meV}$ ). In each case, a zoom of the dashed region is included, as is a momentum-distribution line cut in green for $E=E_{F}$. In (a), the zoom panel clearly shows the $X$ states crossing $E_{F}$, and the same states seen as the outer two features in (b) are joined by an enigmatic, third feature centered on the zone center dubbed the $\Gamma$ state. (c) Data along the same direction as that in (b) but now recorded for $k_{z}=4.5 \pi / a(h \nu=34 \mathrm{eV})$. It is evident that these $\mathrm{Sm} 5 d$-dominated states now have an inverted dispersion with respect to (b). Plus, both the Fermi surface and the 350-meV energy contour (both integrated over $\pm 10 \mathrm{meV}$ ) possess significant intensity around the $\bar{\Gamma}$ point, rather than centered on the $\bar{X}$ point. A schematic of the surface Brillouin zone is added between (c) and (d), showing the color-coded $k$-space coverage of the constant-energy surfaces and the $k$-space trajectories, giving the $E(k)$ images as dashed lines. Finally, (d) illustrates the dichroic signature of the Sm $5 d$-related bands, shown for different binding-energy regimes and $k_{z}$ values. Plotted is the difference in ARPES intensity between data recorded using left or right circular light. It is clear that this dichroic signal has the same sign at all energies below $1 \mathrm{eV}$. In particular, the fact that the same dichroic response is seen for high binding energy (left panel, $h \nu=34 \mathrm{eV}, k_{z}=$ $4.5 \pi / a$ ) and for the $20 \mathrm{meV}$ closest to the Fermi level (right panel, $h \nu=21 \mathrm{eV}, k_{z}=2 \pi / a$ ) shows that the states that give rise to the elliptical Fermi surfaces around the $\bar{X}$ points are no different than those that make up the large-scale $5 d$-related dispersive features pointed out in Fig. 1(d). 
zoomed panels of Figs. 3(a) and 3(b) in a moment, but first we discuss the data shown in Figs. 3(c) and 3(d).

The data in Fig. 3(c) have been acquired with the same sample orientation as those of Fig. 3(b), but with $h \nu=$ $34 \mathrm{eV}$. This photon energy corresponds to a $k_{z}$ value of $4.5 \pi / a$ (see Fig. S1). If there were no dependence of the $E\left(k_{x}, k_{y}\right)$ values of the $\operatorname{Sm} 5 d$-dominated states on $k_{z}$, as would be the case for a surface state, the dispersion relations shown in Figs. 3(b) and 3(c) should be indistinguishable, which is obviously not the case.

Figure 3(c) also shows two constant-energy surfaces (upper at $E_{F}$, lower at $350 \mathrm{meV}$ ). Both reinforce the conclusion that the electronic states-now also those at and close to the Fermi level-for a $k_{z}$ value of $4.5 \pi / a$ do not form elliptical contours around the $\bar{X}$ points as for data acquired at $k_{z}=6 \pi / a[h \nu=70 \mathrm{eV}$; see Figs. 1(d), 2, and $3(\mathrm{a})]$ and $k_{z}=4 \pi / a\left(h \nu=21 \mathrm{eV}\right.$; see Fig. S1). For $k_{z}=$ $4.5 \pi / a$, the momentum distributions are, in fact, centered around $\bar{\Gamma}$, rather than around $\bar{X}$. After a careful inspection of the constant-energy panels of Figs. 2 and 3(c), one can readily conclude that - on one hand - the band topography at $E_{F}$ changes substantially with $k_{z}$, while-on the other hand-there are no major changes between the momentum distributions of the near- $E_{F}$ states and those of the Sm $5 d$ bulk bands acquired at higher $E_{b}$ and the same $k_{z}$. Therefore, by presenting the complete band topography, we can confirm both the non-negligible bulk dispersion of the metallic features and their Sm $5 d$ origin.

Turning now to Fig. 3(d), we offer a final argument for the essentially bulk, Sm $5 d$ character of the $X$ states both at higher energy and near $E_{F}$. Figure 3(d) uses data acquired along $\overline{M X M}$ using circularly polarized light, in which the difference of the photoemission signals acquired with circular left and circular right polarization is shown. The left-hand image $(h \nu=34 \mathrm{eV})$ shows a clear dichroism in intensity [32] between the left and right branches of the $\mathrm{Sm}$ $5 d$-related, strongly dispersive feature already described in the context of Fig. 3(c). The right-hand image is a zoom-in, showing only the first $20 \mathrm{meV}\left(h \nu=21 \mathrm{eV}, k_{z}=4 \pi / a\right)$. These data demonstrate identical dichroism behavior for the high-energy, $\mathrm{Sm} 5 d$-derived states (for example, for $E_{B}=$ $430 \mathrm{meV}$ [see Fig. 2(n)]) and the $X$ states within $20 \mathrm{meV}$ of $E_{F}$. This fact would suggest that they possess the same or very similar orbital character, as the polarization dependence of the photoemission matrix element is sensitive to the symmetry of the initial (and final) states involved [37-39]. This interpretation is backed up by the lack of dichroism at $E\left(k_{x}, k_{y}\right)$ locations where the flat bands have essentially pure $\mathrm{Sm} 4 f$ character-i.e., where the bands strongly differ in orbital character from the $X$ states.

The electronic states relevant for connection to the transport data on $\mathrm{SmB}_{6}$ that are presently creating such excitement are-of course - those very close the Fermi level. The zoomed $E(k)$ images for the $\overline{M X M}$ and $\overline{X \Gamma X}$ directions in Figs. 3(a) and 3(b) directly address the states very close to $E_{F}$. In both cases, there is a swathe of high intensity between binding energies of 40 and $20 \mathrm{meV}$, related to the flat bands connected to the $\mathrm{Sm} 4 f{ }^{6} \mathrm{H}_{5 / 2}$ state. The Sm $5 d$-related $X$ states are visible entering the high-intensity strip from below and leaving it approaching the Fermi level. Comparing the $X$-state $k$ values at higher and lower $E_{B}$, the $k$ discontinuities in the $X$ states are very evident, signaling the strong $d-f$ hybridization, which in turn is consistent with the attribution of the highintensity strip between 20 - and $40-\mathrm{meV}$ binding energy to the $4 f$ states.

These same zoomed images, although a powerful argument for $d-f$ hybridization, also make it very clear that our experiment is not able to resolve the hybridization gaps that must accompany the $k$-renormalization phenomenon. What these data can pin down without any doubt is the fact that the chemical potential in the (near-surface) region probed by ARPES from a superstructure-free, (001) terminated, high-quality $\mathrm{SmB}_{6}$ crystal is not in the $\mathrm{Sm}$ $5 d-\mathrm{Sm} 4 f^{6} \mathrm{H}_{5 / 2}$ hybridization gap, but that this hybridization zone lies some $20 \mathrm{meV}$ below the Fermi level. This discrepancy reveals an overestimation of the hybridizationenergy gaps in LDA calculations $[17,18]$.

To put the similarities and differences between our experimental data and typical theoretical predictions into sharp focus, in Figs. 4(a) and 4(b), we illustrate the changes that can be expected to the dispersion relation of the Sm $5 d$ and $4 f$ states near $E_{F}$ for the two highsymmetry directions probed experimentally in Figs. 3(a) and $3(b)$, when the hybridization interaction is turned off or on. In each panel, the right-hand part (positive $k$ scales) illustrates the nonhybridized situation using solid purple lines. Using the bulk LDA + Gutzwiller theory results of Lu et al. [18] as a basis, we sketch the steep Sm $5 d$ band and three different, much flatter $4 f$ bands. In the left-hand parts (negative $k$ scales), the solid green $(\overline{M X M})$ and orange $(\overline{X \Gamma X)}$ lines show the situation after the hybridization is switched on [18]. The hybridized bands are copied into the right-hand parts of both Figs. 4(a) and 4(b), making it clear that the hybridization gaps open up where the $5 d$ and $4 f$ bands of identical symmetry $\left(\Gamma_{7}[18]\right)$ cross one another.

In the lowest two panels-Figs. 4(c1) and 4(d)- the same illustrative theory curves (hybridized case only) are superimposed on the relevant ARPES $E\left(k_{x}, k_{y}\right)$ images. Two modifications have been made to the theory curves in Fig. 4 compared to those in Ref. [18]: The theory has been shifted $20 \mathrm{meV}$ to a higher binding energy to account for the position of the chemical potential in the ARPES experiment, and the total bandwidth of the ${ }^{6} \mathrm{H}_{5 / 2} 4 f$ multiplet has been rescaled to match the experimental data.

What is clear from the comparison shown in Figs. 4(c1) and $4(\mathrm{~d})$ is that after using the $\mathrm{Sm} 4 f$ states to pin the energy scale of the theory, the rest of the strongly dispersive $d$ - $f$ hybridized bulk bands (that have dominant $d$ 

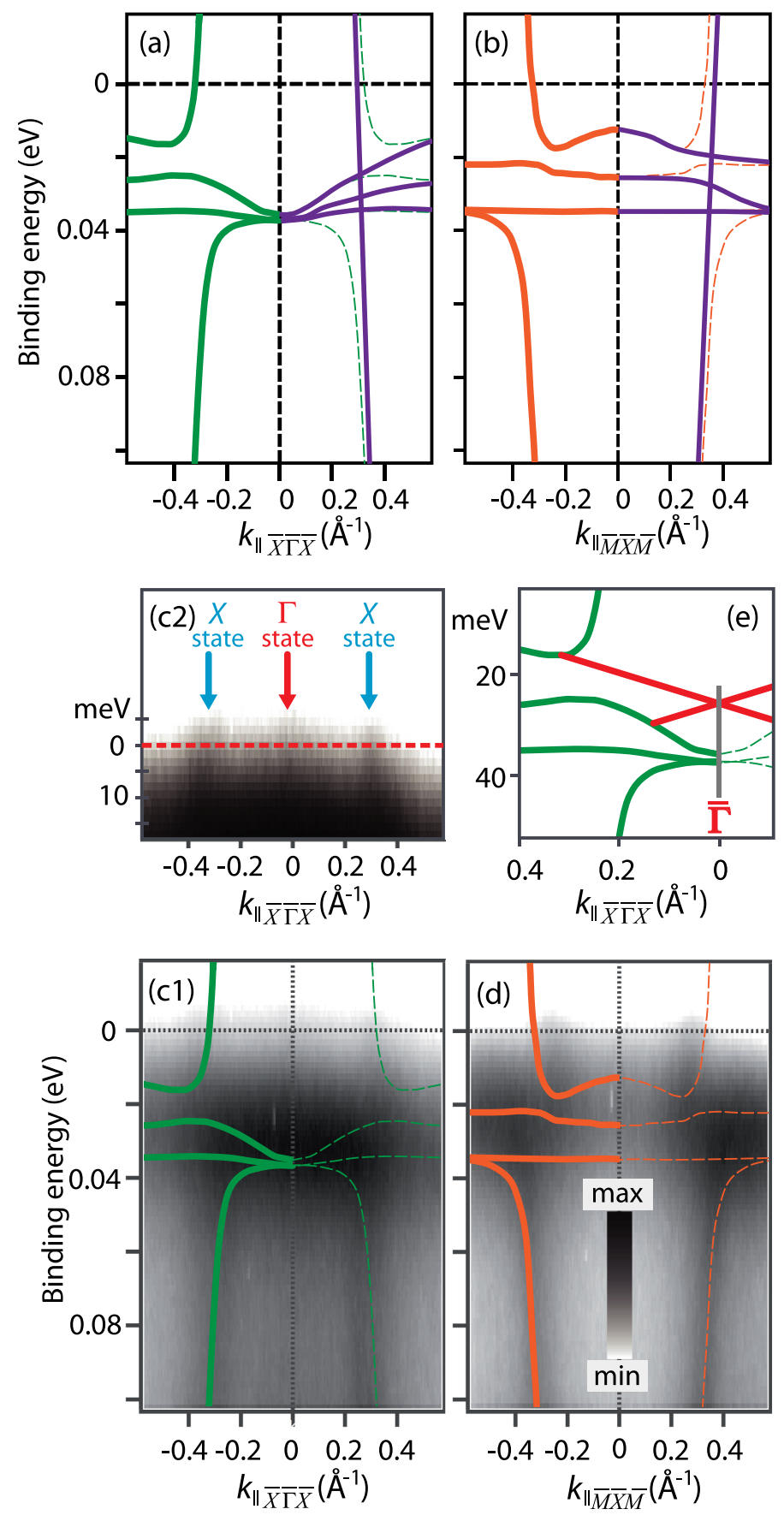

FIG. 4. $k$ discontinuities, position of the chemical potential, and hybridization renormalization from comparison with bulk, ab initio theory. (a),(b) Solid lines in the left (right) parts illustrate hybridized (nonhybridized) $d$ and $f$ bands along two high-symmetry directions in $k$ space. The lines are adaptations of the bulk LDA + Gutzwiller theory data of Ref. [18]. The hybridized cases are overlaid as thin dashed lines in the right parts to aid identification of the hybridization gapping. (c1), (d) The same theory curves are now overlaid on the experimental band structure along $\overline{X \Gamma X}$ and $\overline{M X M} ; k_{z}=6 \pi / a(h \nu=70 \mathrm{eV})$. A very good match is obtained when the total bandwidth of the theoretical $4 f$ multiplet is renormalized by a factor of one-third compared to the original theory data [18], with a simultaneous rigid shift of the theoretical data $20 \mathrm{meV}$ to higher binding energy, carried out to account for a difference in chemical potential between theory and experiment. This renormalization means the $5 d-4 f$ hybridization gaps are only 5-10 meV. From (c1) and (d), it is clear that the $X$ states, including the $k$ discontinuity that is evident on comparing below and above the hybridization zone, are described very well by the energy-scaled bulk theory, removing them from the suspect lineup as far as topological surface states go. In contrast, the $\Gamma$ states, which are highlighted in the zoomed portion of the $\overline{X \Gamma X}$ data in (c2), are missing in the bulk theory. The absence of $\Gamma$ states in bulk calculations is a signal of their promise in terms of identifying potential topological surface states in future ultrahigh-resolution ARPES experiments. (e) A zoom of the theory around $\bar{\Gamma}$, together with a sketch of a massless Dirac cone centered at the $\bar{\Gamma}$ point as a guide for future experiments. 
character) fall nicely on top of the experimental features without any adjustment, confirming our assignment of the $X$ states made earlier on the basis of the $k$ discontinuity in their electronic dispersion relation.

Inspecting the correctly energy-scaled theory curves shown in Fig. 4, it is immediately clear that the hybridization gaps within which possible topologically protected edge states could be situated would be (a) of the order of $20 \mathrm{meV}$ below the Fermi level for the experimentally determined chemical potential and (b) would be only 5-10 meV along both the $\overline{X M}$ and $\overline{\Gamma X}$ high-symmetry directions. Resolving such very small hybridization gaps between the $5 d$ - and $4 f$-derived levels so as to be able to identify a massless Dirac cone as an in-gap state is simply beyond the experimental resolution of the present or recent ARPES studies on $\mathrm{SmB}_{6}$ [30-33].

The comparison shown in Figs. 4(c1) and 4(d) is able to explain all the observed features in the experimental data with the results of bulk hybridization calculations with the exception of what we call the $\Gamma$ state, most clearly seen at the Fermi level for $k=0$ in the data of Fig. 4(c1) and the related zoom-in [Fig. 4(c2)]. This state has not been predicted in bulk calculations [17,18,31].

What is the profile of this as-yet unexplained $\Gamma$ state? First, as mentioned in the discussion of the data of Figs. 3(b) and 3(c), it shows up as increased intensity centered at and near the zone center. The data presented in Figs. 2(f) and 2(g) show that this zone-center intensity is even more pronounced for energies in the middle of the hybridization zones. Such pronounced intensity in the middle of hybridization zones would strongly suggest that the $\Gamma$ state is related to $5 d-4 f$ hybridization and therefore to the opening of Kondo-derived energy gaps.

Interestingly, slab calculations carried out within an LDA + Gutzwiller approach [18] have predicted surface states of topological character within these gaps both at $\bar{X}$ and $\bar{\Gamma}$. In Fig. 4(e), we provide a sketch, showing a zoomed portion of the theory curves for the hybridized states around $\bar{\Gamma}$ (in green) and a Dirac cone at the zone center. Consideration of Figs. 4(c2) and 4(e) together would suggest that the $\Gamma$ state would be the most promising candidate for an experimental indication for the possible existence of topological states. As expected, the ARPES intensity from the $\Gamma$ state fades away as one moves away from the hybridization zones (Fig. 2). However, it is still nonnegligible at $E_{F}$, an issue that could be explained by the superposition of electronic band structures with a different chemical potential coming from a dominant (i.e., $1 \times 1$ ) and a minority atomic arrangement [40]. Despite the high level of clarity of the ARPES data from $\mathrm{SmB}_{6}$ presented here, the weakness and associated indistinctness of the $\Gamma$ state in either our or others' ARPES data made public to date preclude an unambiguous determination of the linear $E\left(k_{x}, k_{y}\right)$ relation for this state and whether it is independent of $E\left(k_{z}\right)$. These observations would form the first steps toward an ARPES-based conclusion on whether $\mathrm{SmB}_{6}$ is a TKI.

We now take a step back from the microscopics of the ARPES data and the very strenuous conditions that will need to be met in future ARPES experiments on $\mathrm{SmB}_{6}$ in order to positively identify topological edge states. We close by summarizing what has been discovered from the data presented here and then discuss how that fits into the larger picture of $\mathrm{SmB}_{6}$.

Our data have established the presence of two different types of states crossing the Fermi level in unreconstructed, high-quality crystals of $\mathrm{SmB}_{6}$.

The first, the $X$ states, possess all the characteristics of bulk Sm $5 d$-derived bands, forming elliptical constantenergy contours for energies away from the $5 d-4 f$ hybridization zones. This assignment is based upon the following facts.

(a) The $X$ states exhibit clear sensitivity to $k_{z}$, belying their significant 3D character.

(b) The experimentally determined Fermi surface of $\mathrm{SmB}_{6}$ does not lie in such a hybridization zone but $20 \mathrm{meV}$ above the energy of the $4 f^{6} \mathrm{H}_{5 / 2}$ multiplet and thus is dominated by the elliptical contours of the $X$ states. In addition, the dichroic signature of the low-lying $X$ states is the same as that for the $5 d$ band at energies well away from both $E_{F}$ and hybridization zones.

(c) The $k$-space signature of the lowest-lying $5 d-4 f$ ${ }^{6} \mathrm{H}_{5 / 2}$ hybridization zone within $20 \mathrm{meV}$ of $E_{F}$ is essentially identical to that of the next hybridization zone involving the $4 f^{6} \mathrm{H}_{7 / 2}$ level at a binding energy of $170 \mathrm{meV}$.

The conclusion just made that the $X$ states that cross $E_{F}$ are not in-gap states is in line with the temperaturedependent ARPES data shown in Refs. [32,33], where what we have identified as the $X$ states and Sm $5 d$ bands merge when $T$ is higher than approximately $100 \mathrm{~K}$. In keeping with experimental determination of Sm valence fluctuations [8] and spectroscopic data [6,7,23], this temperature value is within the regime that marks the onset of appreciable Kondo hybridization. The same $T$-dependent ARPES data [32,33] also suggest that no significant changes happen in the electronic band structure as $T$ decreases from the $38 \mathrm{~K}$ used in this study to $10 \mathrm{~K}$, as both temperatures are well into the Kondo regime [6-8,10,23].

The second type of state we have clearly identified is the $\Gamma$ state, located at or close to the center of the Brillouin zone. These states are maximal in intensity in the hybridization zones between the $5 d$ and $4 f$ states, and are missing from all theory calculations for the bulk of $\mathrm{SmB}_{6}$, making them candidates for possible topological edge states.

Over and above the clear identification of these two main groups of frontier electronic states, our ARPES data have unambiguously proven the following. 
(i) The predicted hybridization gaps of $10-15 \mathrm{meV}$ [18,31] are, in fact, overestimations, rather than underestimations, as stated in previous ARPES studies [6,7,30-33]. We estimate the true hybridization gaps in the $5 d-4 f^{6} \mathrm{H}_{5 / 2}$ hybridization zone to be significantly less than $10 \mathrm{meV}$.

(ii) The experimentally determined location of the chemical potential for the states near the (001) surface is some $20 \mathrm{meV}$ above the lowest-lying $5 d-4 f \quad{ }^{6} \mathrm{H}_{5 / 2}$ hybridization zone, rather than centered within it as theory predicts.

Theory has predicted that the topological surface states of cubic TKIs such as $\mathrm{SmB}_{6}$ should be characterized by heavy effective masses [17]. Such heavy effective masses are in stark contrast to both the light, graphenelike effective masses we observe for the $X$ states (and what we see of the $\Gamma$ state) as well with recent magnetotransport data, which report two distinct Fermi-surface signals with light effective masses as candidates for the topological surface states [21]. The magnetotransport frequencies correspond to Fermi-contour areas of $0.2 \%$ ( $\alpha$ state) and $1.23 \%$ ( $\beta$ state) of the Brillouin zone, which are clearly smaller than the $X$-state electron pockets, which we survey to cover $33 \%$ of the Brillouin zone. In addition, the clearest of the two resonances (the $\beta$ state) is stated to originate from the (101) surface of the $\mathrm{SmB}_{6}$ crystal [21]. The speculation that the $\alpha$ state seen in magnetotransport is related to the (001) plane [21] is at least in line with our finding that the hybridization gaps in which such a topological surface state would live at the (001) surface are only 5-10 meV: A high-Fermi-velocity Dirac cone squeezed into a gap of $5 \mathrm{meV}$ would have to have a very small maximal cross section.

Strong electron correlations have been previously reported to be at the origin of a strong renormalization of the LDA-predicted Sm $4 f$ bandwidth with respect to experimental observations [30]. In line with these results, a later study proposed that LDA + Gutzwiller calculations, a theoretical tool developed for correlated electron systems, can better describe the electronic band structure of $\mathrm{SmB}_{6}$ [18]. We have chosen these theoretical data for comparison with the experimental band structure (Fig. 4), but, as already mentioned, a further renormalization is unavoidable to obtain a satisfactory agreement with the experiment. A comparison of theoretical calculations and ARPES results on similar divalent hexaborides has also identified several discrepancies on gap sizes and bandwidths [41]. Moreover, for the same compounds, it is common that the experimental position of the surface chemical potential lies in the cation $d$-derived conduction band instead of in the band gap between the conduction band and the boron $p$ bands, as proposed theoretically [41]. As a result, electron pockets forming elliptical contours around $X$ have been reported for $\mathrm{EuB}_{6}$ and $\mathrm{SrB}_{6}$, very similar to the Fermi-surface $X$-state contours shown in Figs. 2 and 3 of this work and in other recent ARPES studies of $\mathrm{SmB}_{6}$ [31-33]. Chemical-potential shifts in divalent hexaborides have been attributed to the formation of boron vacancies, with the area of the $\bar{X}$ contours being related to their density at the surface [41]. In the case of $\mathrm{SmB}_{6}$, the effect of stoichiometry on the energy position of the chemical potential has been investigated in an early transport study, where it was concluded that defects such as Sm vacancies could shift the Fermi energy away from the gap value [11]. Therefore, the discrepancies between theoretical predictions and the ARPES results presented here on the hybridization-gap magnitude and the position of the chemical potential fit with other hexaboride data and provide a natural explanation of the ARPES results on $\mathrm{SmB}_{6}(001)$.

\section{CONCLUDING REMARKS}

In the preceding discussion, key facts have been established as regards the character and origin of the low-lying electronic states observed in ARPES from ultrahighvacuum (UHV) cleaved (001) surfaces of $\mathrm{SmB}_{6}$. We now return to the observation at the core of this research field, namely, the unconventional behavior of the resistivity vs temperature for $\mathrm{SmB}_{6}$, which is accepted to be a bulk Kondo insulator.

The transport data on cooling below $100 \mathrm{~K}$ show increasing resistance, valence fluctuations are seen by bulk spectroscopic probes [8], and both our (Fig. 2) and other [32,35] ARPES data possess the telltale signs of $d-f$ hybridization. However, the ARPES data reported here clearly also show the $X$ states at the surface of UHV-cleaved $\mathrm{SmB}_{6}(001)$ to be metallic, thereby creating a dichotomy with the gapped Kondo-insulating state in the bulk. One resolution is to suggest that the $X$ states are topological surface states, living in the Kondohybridization gap [31-33]. Our ARPES data render this explanation untenable, in keeping with the Fermi-surface areas extracted from recent quantum-oscillation data [21]. The key here is the realization that the chemical potential sensed in an ARPES experiment, i.e., that at the surface and the near-surface regions, could be different than that in the bulk, Kondo-insulating state. Our ARPES data indeed show the Fermi level to be approximately $20 \mathrm{meV}$ above the lowest-lying $d$ - $f$ hybridization gap, whereas in the bulk Kondo insulator, $E_{F}$ is within the gap. The picture sketched above would result in a two-conduction-channel scenario (one bulk, one surface), which could explain the observed $T$ dependence of the resistivity [23].

Naturally, the question now arises as to whether there is room at all for the topologically nontrivial surface states consistently predicted by theory for stoichiometric $\mathrm{SmB}_{6}$ ? We answer with reference to the $\Gamma$ states, seen clearly in our ARPES data in the Fermi-surface map of Fig. 3 and also in Fig. 4(c2). These states are strongest in the hybridization gaps and are centered at $\bar{\Gamma}$, just as predicted from theory for the topological surface states $[17,18]$. 
Thus, were the surface of $\mathrm{SmB}_{6}$ to share the same midgapchemical potential as the bulk, then at sufficiently low temperature, the $\Gamma$ states would be the only metallic states and could cause the saturation of the resistivity, possibly exhibiting hallmarks of topological character in magnetotransport experiments [21]. An ARPES proof that the $\Gamma$ states of $\mathrm{SmB}_{6}(001)$ are indeed topological in nature will require the resolution of a massless dispersion relation and spin-momentum locking for a Dirac cone of states squeezed into a hybridization gap of order $5 \mathrm{meV}$. Such energy resolution is beyond the reach of current ARPES instrumentation.

After completion of this paper, a new ARPES + LDA study agreed with our conclusion of the essentially bulk nature of the $X$ states in UHV-cleaved $\operatorname{SmB}_{6}(001)$ [42]. In addition, the discussion of polarity-induced effects [40,42], also coupled to chemical-potential shifts [40], is consistent with the scenario from our ARPES data sketched above.

\section{METHODS}

\section{A. Sample growth and preparation of clean surfaces}

$\mathrm{SmB}_{6}$ single crystals are grown in an optical floatingzone furnace (Crystal Systems, Inc., FZ-T-12000-S-BUPC) under 5-MPa pressure of high-purity argon gas [43]. The growth rate is $20 \mathrm{~mm} / \mathrm{h}$ with the feed and seed rods counterrotating at $30 \mathrm{rpm}$. Samples are cleaved at $38 \mathrm{~K}$ at a pressure lower than $3.0 \times 10^{-10}$ mbar.

\section{B. Angle-resolved photoelectron spectroscopy}

ARPES experiments are performed at the UE112PGM-2a- $1^{2}$ beam line (BESSY II storage ring at the Helmholtz Zentrum Berlin) using a Scienta R8000 hemispherical electron analyzer and a six-axis manipulator. The pressure during measurements is $1.0 \times 10^{-10} \mathrm{mbar}$, while the sample temperature is maintained at $38 \mathrm{~K}$, well into the Kondo regime [6-8,10,23]. The energy position of the Fermi level is determined for every cleave by evaporation of $\mathrm{Au}$ films onto the sample holder, in direct electrical contact with the crystal. Measurements have been acquired with the [100] direction of the sample oriented along the analyzer slit (i.e., $k_{y}$ in Figs. 2 and 3), while the whole Brillouin zone is spanned by sequential rotation of the polar angle. The conversion of the photon energy to the relevant $k_{z}$ values is carried out assuming free-electron final states and an inner potential $V_{o}$ of $16 \mathrm{eV}$ (Supplemental Fig. S1). Our $k_{z}$ values are in good agreement with those of Refs. [30,32,33,35].

\section{ACKNOWLEDGMENTS}

This work is part of the research programme of the Foundation for Fundamental Research on Matter (FOM), which is part of the Netherlands Organization for Scientific
Research (NWO). In addition, we are grateful to F. Massee and M. Grioni for insightful comments.

[1] P. S. Riseborough, Heavy Fermion Semiconductors, Adv. Phys. 49, 257 (2000).

[2] T. Takabatake, F. Iga, T. Yoshino, Y. Echizen, K. Katoh, K. Kobayashi, M. Higa, N. Shimizu, Y. Bando, G. Nakamoto, H. Fujii, K. Izawa, T. Suzuki, T. Fujita, M. Sera, M. Hiroi, K. Maezawa, S. Mock, H. v. Löhneysen, A. Brückl, K. Neumaier, and K. Andres, Ce- and Yb-Based Kondo Semiconductors, J. Magn. Magn. Mater. 177-181, 277 (1998).

[3] G. Aeppli and Z. Fisk, Kondo Insulators, Comments Condens. Matter Phys. 16, 155 (1992).

[4] H. Tsunetsugu, M. Sigrist, and K. Ueda, The Ground-State Phase Diagram of the One-Dimensional Kondo Lattice Model, Rev. Mod. Phys. 69, 809 (1997).

[5] A. Menth, E. Buehler, and T. H. Geballe, Magnetic and Semiconducting Properties of $\mathrm{SmB}_{6}$, Phys. Rev. Lett. 22, 295 (1969).

[6] S. Souma, H. Kumigashira, T. Ito, T. Takahashi, and S. Kunii, Direct Observation of Pseudogap of $\mathrm{SmB}_{6}$ Using Ultrahigh-Resolution Photoemission Spectroscopy, Physica (Amsterdam) 312-313B, 329 (2002).

[7] S. Nozawa, T. Tsukamoto, K. Kanai, T. Haruna, S. Shin, and S. Kunii, Ultrahigh-Resolution and Angle-Resolved Photoemission Study of $\mathrm{SmB}_{6}$, J. Phys. Chem. Solids 63, 1223 (2002).

[8] M. Mizumaki, S. Tsutsui, and F. Iga, Temperature Dependence of $\mathrm{Sm}$ Valence in $\mathrm{SmB}_{6}$ Studied by X-Ray Absorption Spectroscopy, J. Phys. Conf. Ser. 176, 012034 (2009).

[9] T. Kasuya, Gap State in $\mathrm{YbB}_{12}$ and $\mathrm{SmB}_{6}$ : Real Kondo Insulators, Europhys. Lett. 26, 277 (1994).

[10] J. C. Cooley, M. C. Aronson, Z. Fisk, and P. C. Canfield, $\mathrm{SmB}_{6}$ : Kondo Insulator or Exotic Metal?, Phys. Rev. Lett. 74, 1629 (1995).

[11] J. W. Allen, B. Batlogg, and P. Wachter, Large LowTemperature Hall Effect and Resistivity in Mixed-Valent $\mathrm{SmB}_{6}$, Phys. Rev. B 20, 4807 (1979).

[12] J. Derr, G. Knebel, D. Braithwaite, B. Salce, J. Flouquet, K. Flachbart, S. Gabáni, and N. Shitsevalova, From Unconventional Insulating Behavior towards Conventional Magnetism in the Intermediate-Valence Compound $\mathrm{SmB}_{6}$, Phys. Rev. B 77, 193107 (2008).

[13] J.C. Nickerson, R. M. White, K. N. Lee, R. Bachmann, T. H. Geballe, and G. W. Hull, Jr., Physical Properties of $\mathrm{SmB}_{6}$, Phys. Rev. B 3, 2030 (1971).

[14] T. Nanba, H. Ohta, M. Motokawa, S. Kimura, S. Kunii, and T. Kasuya, Gap State of $\mathrm{SmB}_{6}$, Physica (Amsterdam) 186-188B, 440 (1993).

[15] S. Curnoe and K. A. Kinoin, Electron Self-Trapping in Intermediate-Valent $\mathrm{SmB}_{6}$, Phys. Rev. B 61, 15714 (2000).

[16] M. Dzero, K. Sun, V. Galitski, and P. Coleman, Topological Kondo Insulators, Phys. Rev. Lett. 104, 106408 (2010). 
[17] V. Alexandrov, M. Dzero, and P. Coleman, Cubic Topological Kondo Insulators, arXiv:1303.7224.

[18] F. Lu, J. Zhao, H. Weng, Z. Fang, and X. Dai, Correlated Topological Insulators with Mixed Valence, Phys. Rev. Lett. 110, 096401 (2013).

[19] D. J. Kim, S. Thomas, T. Grant, J. Botimer, Z. Fisk, and J. Xia, Robust Surface Hall Effect and Nonlocal Transport in $\mathrm{SmB}_{6}$ : Indication for an Ideal Topological Insulator, arXiv:1211.6769.

[20] D. J. Kim, J. Xia, and Z. Fisk, Topological Surface State in the Kondo Insulator Samarium Hexaboride, arXiv:1307.0448.

[21] G. Li, Z. Xiang, F. Yu, T. Asaba, B. Lawson, P. Cai, C. Tinsman, A. Berkley, S. Wolgast, Y. S. Eo, D.-J. Kim, C. Kurdak, J. W. Allen, K. Sun, X. H. Chen, Y. Y. Wang, Z. Fisk, and L. Li, Quantum Oscillations in Kondo Insulator $\mathrm{SmB}_{6}$, arXiv:1306.5221.

[22] S. Wolgast, C. Kurdak, K. Sun, J. W. Allen, D.-J. Kim, and Z. Fisk, Low Temperature Surface Conduction in the Kondo Insulator $\mathrm{SmB}_{6}$, arXiv:1211.5104.

[23] X. Zhang, N. P. Butch, P. Syers, S. Ziemak, R. L. Greene, and J. Paglione, Hybridization, Inter-Ion Correlation, and Surface States in the Kondo Insulator $\mathrm{SmB}_{6}$, Phys. Rev. X 3, 011011 (2013).

[24] M.Z. Hasan and C..L. Kane, Colloquium: Topological Insulators, Rev. Mod. Phys. 82, 3045 (2010).

[25] M. Bianchi, D. Guan, S. Bao, J. Mi, B. B. Iversen, P. D. C. King, and Ph. Hofmann, Coexistence of the Topological State and a Two-Dimensional Electron Gas on the Surface of $\mathrm{Bi}_{2} \mathrm{Se}_{3}$, Nat. Commun. 1, 128 (2010).

[26] P.D. C. King, R. C. Hatch, M. Bianchi, R. Ovsyannikov, C. Lupulescu, G. Landolt, B. Slomski, J. H. Dil, D. Guan, J. L. Mi, E. D. L. Rienks, J. Fink, A. Lindblad, S. Svensson, S. Bao, G. Balakrishnan, B. B. Iversen, J. Osterwalder, W. Eberhardt, F. Baumberger, and $\mathrm{Ph}$. Hofmann, Large Tunable Rashba Spin Splitting of a Two-Dimensional Electron Gas in $\mathrm{Bi}_{2} \mathrm{Se}_{3}$, Phys. Rev. Lett. 107, 096802 (2011).

[27] M. S. Bahramy, P. D. C. King, A. de la Torre, J. Chang, M. Shi, L. Patthey, G. Balakrishnan, Ph. Hofmann, R. Arita, N. Nagaosa, and F. Baumberger, Emergent Quantum Confinement at Topological Insulator Surfaces, Nat. Commun. 3, 1159 (2012).

[28] C. Nayak, S. H. Simon, A. Stern, M. Freedman, and S. Das Sarma, Non-Abelian Anyons and Topological Quantum Computation, Rev. Mod. Phys. 80, 1083 (2008).

[29] J. G. Analytis, J.-H. Chu, Y. Chen, F. Corredor, R. D. McDonald, Z.-X. Shen, and I. R. Fisher, Bulk Fermi Surface Coexistence with Dirac Surface State in $\mathrm{Bi}_{2} \mathrm{Se}_{3}$ : A Comparison of Photoemission and Shubnikovde Haas Measurements, Phys. Rev. B 81, 205407 (2010).

[30] H. Miyazaki, T. Hajiri, T. Ito, S. Kunii, and S.-i. Kimura, Momentum-Dependent Hybridization Gap and Dispersive In-Gap State of the Kondo Semiconductor $\mathrm{SmB}_{6}$, Phys. Rev. B 86, 075105 (2012).

[31] M. Neupane, N. Alidoust, S.-Y. Xu, T. Kondo, D.-J. Kim, C. Liu, I. Belopolski, T.-R. Chang, H.-T. Jeng, T. Durakiewicz, L. Balicas, H. Lin, A. Bansil, S. Shin, Z.
Fisk, and M.Z. Hasan, Surface Electronic Structure of a Topological Kondo Insulator Candidate $\mathrm{SmB}_{6}$ : Insights from High-Resolution ARPES, arXiv:1306.4634.

[32] J. Jiang, S. Li, T. Zhang, Z. Sun, F. Chen, Z. R. Ye, M. Xu, Q. Q. Ge, S. Y. Tan, X. H. Niu, M. Xia, B. P. Xie, Y.F. Li, X. H. Chen, H. H. Wena, and D. L. Feng, Observation of In-Gap Surface States in the Kondo Insulator $\mathrm{SmB}_{6}$ by Photoemission, arXiv:1306.5664.

[33] N. Xu, X. Shi, P. K. Biswas, C.E. Matt, R. S. Dhaka, Y. Huang, N.C. Plumb, M. Radovic, J.H. Dil, E. Pomjakushina, A. Amato, Z. Salman, D. McK. Paul, J. Mesot, H. Ding, and M. Shi, Surface and Bulk Electronic Structure of the Strongly Correlated System $\mathrm{SmB}_{6}$ and Implications for a Topological Kondo Insulator, Phys. Rev. B 88, 121102(R) (2013).

[34] M. Aono, R. Nishitani, T. Tanaka, E. Bannai, and S. Kawai, Azimuthal Anisotropy in Low-Energy Ion Scattering from $\mathrm{SmB}_{6}$ (001), Solid State Commun. 28, 409 (1978).

[35] J. D. Denlinger, G.-H. Gweon, J. W. Allen, C. G. Olson, Y. Dalichaouch, B.-W. Lee, M. B. Maple, Z. Fisk, P.C. Canfield, and P.E. Armstrong, Advances in Photoemission Spectroscopy of $f$-Electron Materials, Physica (Amsterdam) 281-282B, 716 (2000).

[36] See Supplemental Material at http://link.aps.org/ supplemental/10.1103/PhysRevX.3.041024 for the data movies S2a and S2b describing the evolution of the elliptical contours as a function of binding energy.

[37] C. Westphal, J. Bansmann, M. Getzlaff, and G. Schönhense, Circular Dichroism in the Angular Distribution of Photoelectrons from Oriented CO Molecules, Phys. Rev. Lett. 63, 151 (1989).

[38] A. Kaminski, S. Rosenkranz, H. M. Fretwell, J.C. Campuzano, Z. Li, H. Raffy, W. G. Cullen, H. You, C. G. Olson, C. M. Varma, and H. Höchst, Spontaneous Breaking of Time-Reversal Symmetry in the Pseudogap State of a High- $T_{c}$ Superconductor, Nature (London) 416, 610 (2002).

[39] D. V. Vyalikh, S. Danzenbächer, A. N. Yaresko, M. Holder, Y. Kucherenko, C. Laubschat, C. Krellner, Z. Hossain, C. Geibel, M. Shi, L. Patthey, and S.L. Molodtsov, Photoemission Insight into Heavy-Fermion Behavior in $\mathrm{YbRh}_{2} \mathrm{Si}_{2}$, Phys. Rev. Lett. 100, 056402 (2008).

[40] M. M. Yee, Y. He, A. Soumyanarayanan, D.-J. Kim, Z. Fisk, and J.E. Hoffman, Imaging the Kondo Insulating Gap on $\mathrm{SmB}_{6}$, arXiv:1308.1085.

[41] J.D. Denlinger, J. A. Clack, J.W. Allen, G.-H. Gweon, D. M. Poirier, C. G. Olson, J. L. Sarrao, A. D. Bianchi, and Z. Fisk, Bulk Band Gaps in Divalent Hexaborides, Phys. Rev. Lett. 89, 157601 (2002).

[42] Z.-H. Zhu, A. Nicolaou, G. Levy, N.P. Butch, P. Syers, X. F. Wang, J. Paglione, G. A. Sawatzky, I. S. Elfimov, and A. Damascelli, Polarity-Driven Surface Metallicity in $\mathrm{SmB}_{6}$, arXiv:1309.2945.

[43] L. H. Bao, O. Tegus, J. X. Zhang, X. Zhang, and Y. K. Huang, Large Emission Current Density of $\mathrm{La}_{x} \mathrm{Ce}_{1-x} \mathrm{~B}_{6}$ High Quality Single Crystals Grown by Floating Zone Technique, J. Alloys Compd. 558, 39 (2013). 